\title{
Comparative analysis of healthcare provision to individuals with cleft lip and/or palate at specialised academic centres in South Africa
}

\author{
P Hlongwa, ${ }^{1,2} \mathrm{BOH}, \mathrm{BDS}, \mathrm{MDent}$ (Ortho); T C Dandajena, ${ }^{1} \mathrm{PhD} ; \mathrm{L}$ C Rispel, ${ }^{2} \mathrm{PhD}$ \\ ${ }^{1}$ Department of Orthodontics, School of Oral Health Sciences, Faculty of Health Sciences, University of the Witwatersrand, Johannesburg, South Africa \\ ${ }^{2}$ Centre for Health Policy, School of Public Health, Faculty of Health Sciences, University of the Witwatersrand, Johannesburg, South Africa
}

Corresponding author: P Hlongwa (phumzile.hlongwa@wits.ac.za)

\begin{abstract}
Background. Cleft lip and/or palate (CLP) is the most common congenital anomaly of the craniofacial complex, with an estimated worldwide prevalence of $1 / 500-700$ live births. Affected children require immediate medical treatment and prolonged management by a multidisciplinary team of health professionals.

Objectives. To describe and compare healthcare provision to individuals with CLP at specialised care centres in South Africa (SA).

Methods. The study was conducted at all CLP care centres in 6 of SA's 9 provinces that provide specialised treatment and care to individuals with CLP. At each centre, the team leader was interviewed using a semi-structured questionnaire that focused on the point-of-care entry for CLP patients; type of services provided; whether treatment protocols were used, which treatment protocols were used and internal referral systems; and members of the healthcare team. Stata 13 (StataCorp., USA) was used to analyse the data.

Results. Eleven CLP team leaders participated in the study, of whom 5 were from Gauteng Province. The point-of-care for CLP patients in the majority of centres was plastic surgery $(n=9 / 11 ; 81.8 \%)$. The majority of centres $(n=10 / 11 ; 90.9 \%)$ followed similar treatment protocols and only 1 centre performed lip surgery at 12 - 18 months. Although all centres reported a multidisciplinary team approach for CLP care provision, there were gaps in the health professions categories, which influenced the type of treatment provided. Hence, surgical repair of the lip and palate $(n=10 / 11 ; 90.9 \%)$ and speech therapy $(n=7 / 11 ; 63.6 \%)$ dominated the type of treatment provided, and patients were referred to other provinces or to the private health sector for other types of treatment.

Conclusions. The gaps in services at the CLP care centres in SA need to be addressed to ensure integrated, holistic care provision.
\end{abstract}

S Afr Med J 2019;109(6):426-430. DOI:10.7196/SAMJ.2019.v109i6.13654

Cleft lip and/or palate (CLP) is the most common congenital anomaly of the craniofacial complex, with an estimated worldwide prevalence of $1 / 500-700$ live births. ${ }^{[1]}$ In South Africa (SA), CLP is among the 5 most common birth defects reported, ${ }^{[2]}$ with an estimated prevalence of $0.1-0.4 / 1000$ live births. ${ }^{[2-4]}$

Affected children present with a number of medical problems and potential complications that include feeding difficulties, hearing loss, speech problems, disfigured appearance and dental malformation. ${ }^{[5-8]}$ Hence, individuals with CLP require co-ordinated and specialised treatment offered by a multidisciplinary team, ${ }^{[9-11]}$ which includes geneticists, maxillofacial surgeons, otolaryngologists, orthodontists, paediatricians, plastic surgeons, paediatric dentists, psychologists, professional nurses, social workers and speech and language therapists. ${ }^{[12-14]}$ These teams are available in high-income countries (HICs).

Various clinical protocols have been proposed for the treatment of CLP. ${ }^{[15]}$ Many HICs have adopted national protocols and guidelines and have centralised services and/or established centres for the management and follow-up of affected individuals. ${ }^{[16-19]}$ The advantages of centralisation and/or concentration of specialised services include the following: co-ordination of treatment, standardised data collection, availability of a critical mass of experts, clinical audit and review to enhance quality of care, responsiveness to patient and family needs, and ongoing monitoring and evaluation. ${ }^{[12,20-26]}$

Globally, it is estimated that only $20 \%$ of individuals with CLP have access to comprehensive treatment that involves a team co-ordinated approach. ${ }^{[27]}$ The treatment and care gaps include: lack or shortages of healthcare professionals, lack of infrastructure and delays in surgical repair of the clefts. ${ }^{[27,28]}$ These gaps are most pronounced in low- and middle-income countries (LMICs) in Africa, Asia and South America ${ }^{[28,29]}$ In many LMICs, outreach programmes by nongovernmental organisations (NGOs) have assisted in improving the services for individuals with CLP. ${ }^{[29-34]}$ These NGOs, together with support groups that include parents of the affected children, have played an important role in the ongoing management of CLP patients. However, the majority of LMICs, including SA, lag behind in terms of national treatment protocols, standardised data collection, access to care by a multidisciplinary team, and evaluation of treatment outcomes. ${ }^{[12,18,35]}$

Notwithstanding improvements in the treatment of CLP individuals in the preceding decades, ${ }^{[36-38]}$ there is still no universal protocol for repair and ongoing clinical management. However, treatment modalities in the management of CLP are often based on chronological age and dentofacial development. ${ }^{[38]}$ The common elements of clinical standards and sequence of treatment of CLP are shown in Table $1 .^{[38]}$ At birth, genetic counselling is given to parents, and feeding of the baby is evaluated. Depending on the size of the cleft, feeding plates are recommended within the first week after birth to assist with feeding. Presurgical infant orthopaedic treatment for approximation of the segment is also performed prior to cleft lip repair. As the child grows, several follow-up consultations for management of the cleft are done until treatment is completed during

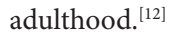




\begin{tabular}{ll} 
Table 1. Treatment sequence in the management of cleft lip and/or palate \\
\hline Chronological age/dentofacial development & Cleft lip and/or palate treatment \\
\hline At birth & Genetic counselling \\
& Feeding plate \\
& Presurgical infant orthopaedics \\
& Psychosocial counselling \\
& Surgical lip repair \\
6 months 12 months & Surgical palate repair \\
& Grommets \\
& Speech therapy \\
& Routine dental treatment \\
$5-7$ years & Alveolar bone graft \\
& Speech therapy \\
& Dental treatment/maxillary expansion/bone graft/dental arch alignment \\
& Speech therapy \\
$10-14$ years & Orthodontic treatment/maxillary expansion/bone graft/maxillary protraction \\
& Orthodontic treatment/orthognathic surgery/maxillary advancement \\
$19-20$ years & Psychology counselling \\
& Prosthodontic replacement of missing teeth \\
Adapted from De Ladeira and Alonso. ${ }^{[38]}$ & Nose revision \\
& \\
&
\end{tabular}

There is a dearth of studies on the management of CLP in SA, except for a 1953 study performed in a Johannesburg private hospital, which found that the clinical management of patients with CLP was uncoordinated. ${ }^{[39]}$ The aim of our study was to compare the treatment and care of individuals with CLP at all the CLP care centres in SA. This is part of a larger doctoral study on the epidemiology and care of individuals with CLP in SA.

\section{Methods}

This cross-sectional study was conducted during 2015 and 2016 in all the 11 specialised, academic CLP care centres in 6 of SA's 9 provinces.

Confidentiality was maintained throughout the study. The same unique identification number was allocated to each centre and CLP team leader to ensure anonymity. The data containing unique numbers and centre information were kept on a password-protected computer.

The CLP team leaders were interviewed using a semi-structured questionnaire that contained 29 questions divided into 3 parts, i.e. centre information, treatment and management of CLP and team members. The questions focused on the entry point of care for CLP individuals, type of services and treatment provided at each centre, treatment protocols followed in the clinical management of individuals with CLP, continuum of care, internal referral systems, as well as healthcare professionals who were members of the team. The interviews were complemented with a record review of all individuals with CLP who visited the academic centres from 1 January 2013 to 31 December 2014.

At each centre, the principal investigator conducted the interviews in a private room. Each interview lasted an average of 30 minutes. The responses of participants were written down verbatim in the space provided in the questionnaire, typed out and saved as individual Microsoft Word documents. The data were imported into Stata 13 (StataCorp., USA) for descriptive analysis.

\section{Ethical approval}

The Human Research Ethics Committee (medical) of the University of the Witwatersrand, Johannesburg, provided ethical approval (ref. no. M150536). Approval was also obtained from the CLP care centres and permission was obtained from the relevant healthcare authorities. We adhered to standard ethical procedures, which included study information sheets, voluntary participation, informed consent, anonymity of responses and confidentiality. The participants' consent was in written form.

\section{Results}

A 100\% response rate was obtained and all 11 team leaders from CLP care centres participated in the study: 5 in Gauteng Province, 2 in Western Cape Province and 1 in each of the provinces of the Eastern Cape, Free State, KwaZulu-Natal and Limpopo. Most of the centres have been in existence for $>5$ years. The descriptive characteristics of the centres are shown in Table 2.

One centre in Gauteng had no records for the 2-year review period. The number of individuals with CLP at the 10 centres totalled 699, ranging from 16 to 144 . The team leaders reported that individuals with CLP were treated primarily by plastic surgeons. Most centres reported a team approach for the provision of CLP treatment.

The treatment provided by each centre is shown in Table 3. The dominant modes of treatment were surgical repair and speech therapy. Other treatment modalities were less common. Although centre 9 is listed as a CLP specialised centre, it does not provide any active treatment but serves as an NGO booking and follow-up centre for surgical repairs.

Six centres provided their treatment protocols for analysis (Table 4 ). The protocols showed that 2 centres used feeding plates and presurgical orthopaedic treatment. All 6 centres followed a set protocol for timing of the surgical procedure for the lip and palate. The sequencing of lip surgery was similar in 5 centres. Palate surgery and alveolar bone graft were performed at similar times in all 6 centres. While centres 4 and 5 reported availability of orthodontic treatment, age of treatment was not indicated. Similarly, orthognathic treatment was performed at 4 centres and speech therapy was offered by all 6 centres; however, no specific age of delivery was indicated. Other treatments were not provided according to the standard protocol (Table 1), were not provided when needed and were not available in most centres. 
Table 5 shows the categories of healthcare professionals (HCP) at each CLP care centre. In most centres, plastic surgeons (91\%) and speech therapists (72.7\%) formed part of the CLP team. Other HCPs,

\begin{tabular}{ll}
$\begin{array}{l}\text { Table 2. Descriptive characteristics of the cleft lip and/or } \\
\text { palate centres }\end{array}$ \\
\hline Characteristics & Sample size, $\boldsymbol{n}$ (\%) \\
\hline Cleft lip and/or palate team leader \\
$(N=11)$ \\
Plastic surgery & $9(81.8)$ \\
Maxillofacial surgery & $1(9.1)$ \\
Orthodontics & $1(9.1)$ \\
Type of care provided $(N=11)$ & \\
Individual specialist & $1(9.1)$ \\
Team approach & $6(54.6)$ \\
Hybrid approach & $3(27.3)$ \\
Other & $1(9.1)$ \\
Cleft lip and/or palate per academic & \\
centre (n=699) & \\
Centre 2 & \\
Centre 3 & $93(13.3)$ \\
Centre 4 & $33(4.7)$ \\
Centre 5 & $52(7.4)$ \\
Centre 6 & $141(20.2)$ \\
Centre 7 & $79(11.3)$ \\
Centre 8 & $70(10.0)$ \\
Centre 9 & $62(8.9)$ \\
Centre 10 & $16(2.3)$ \\
Centre 11 & $122(17.5)$ \\
& $31(4.4)$ \\
&
\end{tabular}

such as ear, nose and throat surgeons, paediatric dentists, paediatric surgeons, psychologists and social workers, were not available in most of the centres. Although professional nurses were present in 7 centres (63.6\%), in only 2 centres were these nurses actively involved with CLP treatment; they played primarily administrative roles in the other 5 centres. Only 2 centres reported that parents' support groups were incorporated in CLP management.

\section{Discussion}

This is one of the first comparative studies to analyse treatment and care of individuals with CLP in the SA public sector. Our study found variations across the 11 care centres in the number of CLP patients treated, type of treatment provided, availability of written protocols and composition of healthcare teams.

The number of individuals with CLP treated during the 2-year period of the review ranged from 16 to 141 . Centre 9 did not meet the criteria for a specialised centre, as it only provided booking and follow-up services to CLP individuals. Experts have pointed to the importance of sufficient case-loads to ensure competent clinical care and to secure adequate resources for comprehensive care ${ }^{[40]}$ A 2001 review in the UK led to a government directive to provide care from a single regional centre, with a comprehensive specialist team and a guideline that 1 surgeon should be responsible for 40 - 50 new patients requiring primary surgery per year. ${ }^{[18,41]}$ Similarly, cleft care has also been centralised in Brazil ${ }^{[2,43]}$ and in the Scandinavian countries of Denmark, Finland, Norway and Sweden. ${ }^{[21]}$ However, the concept of regionalisation or concentration of CLP services is under-developed in SA, exacerbated by the relatively low priority of congenital anomalies within the overall context of the country's quadruple disease burden.

The current study found that the dominant modalities of treatment were surgical repair of the lip and palate ( $n=10 / 11 ; 90.9 \%$ of centres)

Table 3. Types of treatment for cleft lip and/or palate provided by each academic centre $(N=11)$

\begin{tabular}{|c|c|c|c|c|c|c|c|c|c|c|}
\hline \multirow[b]{2}{*}{ Centre } & \multicolumn{10}{|c|}{ Treatment } \\
\hline & FP & PSO & ST & GC & LPR & GDT & ORT & OGT & ABG & ENT \\
\hline 1 & No & No & Yes & Yes & Yes & No & No & No & No & No \\
\hline 2 & No & No & Yes & No & Yes & No & No & No & Yes & No \\
\hline 3 & No & No & No & No & Yes & No & No & No & No & No \\
\hline 4 & Yes & Yes & Yes & No & Yes & Yes & Yes & Yes & Yes & No \\
\hline 5 & Yes & Yes & Yes & Yes & Yes & Yes & Yes & Yes & Yes & No \\
\hline 6 & No & No & Yes & Yes & Yes & No & Yes & Yes & Yes & Yes \\
\hline 7 & No & No & Yes & Yes & Yes & Yes & Yes & No & No & Yes \\
\hline 8 & No & No & Yes & Yes & Yes & Yes & Yes & Yes & Yes & No \\
\hline 9 & No & No & No & No & No & No & No & No & No & No \\
\hline 10 & No & No & Yes & Yes & Yes & No & No & Yes & No & No \\
\hline 11 & No & No & No & No & Yes & No & No & No & No & No \\
\hline Total, $n$ & 2 & 2 & 8 & 6 & 10 & 4 & 5 & 5 & 5 & 2 \\
\hline
\end{tabular}

Table 4. Comparison of cleft lip and/or palate treatment protocols across 6 centres*

\begin{tabular}{llllll}
\hline Centre & Feeding plate & Presurgical orthopaedics & Lip repair, months & Palate repair, months & Alveolar bone graft, years \\
\hline 2 & No & No & 3 & $6-12$ & $6-8$ \\
4 & Birth & Birth & $3-6$ & $12-18$ & $8-10$ \\
5 & Birth & Birth & $12-18$ & $6-12$ & $8-10$ \\
6 & No & No & 3 & $6-12$ & $8-10$ \\
7 & No & No & 3 & $12-18$ & $8-10$ \\
8 & No & No & 3 & 9 & $7-11$ \\
${ }^{*}$ These centres provided written protocols for analysis.
\end{tabular}


Table 5. Members of cleft lip and/or palate team in each academic centre

\begin{tabular}{|c|c|c|c|c|c|c|c|c|c|c|c|c|}
\hline \multirow[b]{2}{*}{ Centre } & \multicolumn{11}{|c|}{ Team members } & \multirow[b]{2}{*}{ PSG } \\
\hline & Gen & PS & Orth & ENT & MFS & PD & Paed & Psych & PN & SW & ST & \\
\hline 1 & 1 & 1 & 0 & 0 & 0 & 0 & 0 & 0 & 1 & 1 & 1 & 1 \\
\hline 2 & 0 & 1 & 0 & 0 & 1 & 0 & 0 & 0 & 1 & 1 & 1 & 0 \\
\hline 3 & 0 & 1 & 0 & 0 & 0 & 0 & 0 & 0 & 1 & 0 & 0 & 0 \\
\hline 4 & 0 & 1 & 1 & 0 & 1 & 1 & 0 & 0 & 0 & 0 & 1 & 0 \\
\hline 5 & 1 & 0 & 1 & 0 & 1 & 1 & 0 & 0 & 1 & 0 & 1 & 1 \\
\hline 6 & 1 & 1 & 1 & 1 & 1 & 0 & 0 & 0 & 1 & 0 & 1 & 0 \\
\hline 7 & 1 & 1 & 1 & 1 & 0 & 1 & 0 & 1 & 0 & 0 & 1 & 0 \\
\hline 8 & 1 & 1 & 1 & 0 & 1 & 1 & 1 & 0 & 1 & 0 & 1 & 0 \\
\hline 9 & 0 & 1 & 0 & 0 & 0 & 0 & 0 & 0 & 1 & 0 & 0 & 0 \\
\hline 10 & 1 & 1 & 0 & 0 & 0 & 0 & 0 & 1 & 0 & 0 & 1 & 0 \\
\hline 11 & 0 & 1 & 0 & 0 & 0 & 0 & 1 & 0 & 0 & 0 & 0 & 0 \\
\hline Total, $n(\%)$ & $6(54.5)$ & $10(91)$ & $5(45.5)$ & $2(18.2)$ & $5(45.5)$ & $4(36.4)$ & $2(18.1)$ & $2(18.2)$ & $7(63.6 \%)$ & $2(18.2)$ & $8(72.7)$ & $2(18.2)$ \\
\hline
\end{tabular}

and speech therapy $(n=7 / 11 ; 63.6 \%)$. Two of the centres that did not provide speech therapy were located in the country's rural provinces, and 1 centre (centre 9) did not provide any active treatment for CLP. This underscores the inequities in access to specialised services in the SA public health sector. ${ }^{[44]}$ Speech therapy is an essential service for CLP. This gap would need to be addressed, either through efforts to recruit speech therapists to these centres, formal referral to and/or partnerships with urban CLP centres, or public/private partnerships.

This study found that feeding plates were underutilised, as 2 centres $(n=2 / 11 ; 18.2 \%)$ reported their use, while the other 9 centres $(n=9 / 11 ; 81.8 \%)$ did not use them for CLP patients. Notwithstanding the controversies regarding the use of feeding plates, ${ }^{[45-47]}$ feeding difficulties following the birth of babies with CLP are common and the feeding plate can assist with closure of the cleft to facilitate sucking and to prevent choking. A study among caregivers of CLP children found that feeding difficulties were one of the most distressing aspects in caring for the children, ${ }^{[48]}$ highlighting the importance of adequate support and assistance to caregivers regarding feeding methods.

The comparison of treatment protocols was only possible for 6 of the 11 centres. Five of the centres did not have a written protocol, which means that care might not be standardised in these centres, which could influence the quality of care provided. CLP care centres should be encouraged to develop standard treatment protocols. The findings of this study could be used to develop standard treatment guidelines, with the involvement of all 11 CLP centres, drawing on the experiences of the centres with protocols and international best practice. ${ }^{[21,42,43]}$

The timing for lip repair was 3 - 6 months in most centres. However, for our sample, the median age at consultation was $\sim 3$ months, with a high interquartile range of 3 weeks - 13 months; i.e. a sizeable portion of these children presented after 6 months, when ideally their first operation should have been done. This delayed age of consultation has also been reported in other LMICs. ${ }^{[49-51]}$ Lip surgery provides comfort to the parents of CLP children, as they are able to show their children in public and avoid stigmatisation. Strategies need to be developed to ensure that the majority of children with CLP have access to lip surgery within the first 6 months of life. This will assist with the reduction of possible physical impairment and societal courtesy stigma and discrimination. ${ }^{[48,52]}$

There was variation in the number of team members at CLP centres, ranging from 2 to 8 . In most centres, there was a scarcity of certain specialists. There are existing guidelines on health professionals who should constitute members of CLP teams. ${ }^{[12]}$ Although the SA context would need to be taken into account, these guidelines should inform the constitution of the multidisciplinary team. Our study found that professional nurses were underutilised as members of the team. In other settings, professional nurses play an important role, especially in assisting mothers with feeding. ${ }^{[12,53,54]}$

The current study revealed that only 2 centres reported incorporating CLP parent support groups in their management. Other studies found that parents' involvement and participation in care decisions are important in CLP care outcomes, as their knowledge and support enhance compliance with treatment, retention and reduction of complications. ${ }^{[48,55,56]}$

\section{Study limitations}

Although our study generated new knowledge on the types of treatment available at all public sector CLP centres, it was largely descriptive. We did not evaluate the following: types of surgery used for treatment, quality of care, outcomes of treatment, cost of treatment and follow-up treatment provided to CLP individuals. These are study limitations, but point to areas for further research. Nonetheless, the knowledge generated by this study can be used to develop standard treatment guidelines for individuals with CLP, enhance the discourse on appropriate treatment modalities and contribute to the development of collaborative partnerships across the 11 centres.

\section{Conclusions}

Although the treatment of children with CLP and other types of craniofacial anomalies in SA has been ongoing for $>6$ decades, ${ }^{[39]}$ comprehensive care is still lacking. The study has shown that there are gaps in the types of treatment provided across the 11 centres, that not all members of the multidisciplinary team are present, and that standardised treatment protocols are absent. These gaps are more pronounced in centres located in the rural provinces of SA.

\section{Recommendations}

The study findings underscore the need for the National Department of Health $(\mathrm{NDoH})$ to review and update the national policy on congenital anomalies, which has not been revised since 2005. The $\mathrm{NDoH}$ should also ensure equitable access to appropriate treatment and care for individuals with CLP in SA, regardless of geographical 
location. This might be achieved through regionalisation of centres and developing a standard treatment protocol for CLP, in consultation and with the involvement of health professionals at these 11 centres. Such regionalisation will ensure the existence of a critical mass of health professionals at each specialist facility, foster collaboration, enhance the education and training of healthcare providers on congenital anomalies, enable sharing of experiences and mutual learning, optimise resource utilisation and facilitate research on treatment models and outcomes. Ultimately, these initiatives will contribute to quality-of-care improvements for all individuals with CLP.

Appendix 1. The dataset supporting the conclusions of this article is not currently available, as it is part of $\mathrm{PH}$ 's $\mathrm{PhD}$ thesis. The questionnaire used to collect the data is available from the corresponding author on request. Declaration. Article based on a study done by PH in partial fulfilment of her $\mathrm{PhD}$ thesis.

Acknowledgements. We thank all participating academic centres for allowing us to review their CLP records.

Author contributions. LCR is the primary supervisor of the $\mathrm{PhD}$ and TCD the co-supervisor. $\mathrm{PH}$ and LCR conceptualised and designed the $\mathrm{PhD}$ study. PH collected all the data, PH and LCR analysed the data. All authors contributed to writing and editing the manuscript, and read and approved the final version.

Funding. The PhD project was funded by the Wits Faculty of Science Research Office; SA Society of Orthodontists Gerald Gavron Fund; and SA Dental Association Dentistry Developmental Foundation of SA Trust. The funding sources were not involved in the design of the study, data collection, analysis, interpretation of results or writing of the manuscript. Conflicts of interest. None.

1. World Health Organization. Addressing the Global Challenges of Craniofacial Anomalies. Report of a WHO Meeting on International Collaborative Research on Craniofacial Anomalies. Geneva: WHO, 2006

2. Kromberg JG, Jenkins T. Common birth defects in South African Blacks. S Afr Med J 1982;62(17):599602 .

3. Morrison G, Cronje AS, van Vuuren I, et al. The incidence of cleft lip and palate in the Western Cape. S Afr Med J 1985;68(8):576-577.

4. Van Wyk PJ, Bütow KW, van der Merwe CA, et al. [Incidence and clinical appearance of cleft deformities in the Transvaal]. J Dent Ass S Afr 1987;42(7):403-406.

5. Camille A, Evelyne AK, Martial AE, et al. Advantages of early management of facial clefts in Africa. Int J Pediatr Otorhinolaryngol 2014;78(3):504-506. https://doi.org/10.1016/j.ijporl.2013.12.031

6. Miller CK. Feeding issues and interventions in infants and children with clefts and craniofacial syndromes. Semin Speech Language 2011;32(2):115-126. https://doi.org/10.1055/s-0031-1277714

7. Kummer AW. Evaluation of speech and resonance for children with craniofacial anomalies. Facial Plast Kummer AW. Evaluation of speech and resonance for children with craniofacial a
Surg Clin North Am 2016;24(4):445-451. https://doi.org/10.1016/j.fsc.2016.06.003

Surg Clin North Am 2016;24(4):445-451. https://doi.org/10.1016/j.fsc.2016.06.003
8. Bell J, Nassar N, Turner R, et al. Hospitalisations up to adulthood for children born with orofacial 8. Bell J, Nassar N, Turner R, et al. Hospitalisations up to adulthood for children bo
clefts. J Paediatr Child Health 2016;52(4):441-448. https://doi.org/10.1111/jpc.13024

clefts. J Paediatr Child Health 2016;52(4):441-448. https://doi.org/10.1111/jpc.13024
9. Kennedy TJ, Miller SH, Tooze FM, et al. The team approach to treatment of the cleft lip and palate. Kennedy TJ, Miller SH, Tooze F

Am Fam Phys 1978;18(1):74-84.
10. Wellens W, vander Poorten V. Keys to a successful cleft lip and palate team. B-ENT 2006;2(Suppl 4):3-10.

11. Akinmoladun VI, Obimakinde OS, Okoje VN. Team approach to management of oro-facial cleft among African practitioners: A survey. Nigerian J Clin Pract 2013;16(1):86-90. https://doi.org/10.4103/11193077.106773

12. American Cleft Palate-Craniofacial Association. Parameters for evaluation and treatment of patients with cleft lip/palate or other craniofacial differences. Cleft Palate Craniofac J 2018;55(1):137-156. https://doi. org $/ 10.1177 / 1055665617739564$

13. Tindlund RS, Holmefford A, Eriksson J-CH, et al. Interdisciplinary evaluation of consecutive patients with unilateral cleft lip and palate at age 6, 15, and 25 years: A concurrent standardized procedure and documentation by plastic surgeon; speech and language pathologist; ear, nose, and throat specialist; and orthodontist. J Craniofac Surg 2009;20(8):1687-1698.

14. Bardach J, Morris H, Olin W, et al. Late results of multidisciplinary management of unilateral cleft lip and palate. Ann Plast Surg 1984;12(3):235-242.

15. Shaw WC, Dahl E, Asher-McDade C, et al. A six-center international study of treatment outcome in patients with clefts of the lip and palate. Part 5: General discussion and conclusions. Cleft Palate Craniofac J 1992;29(5):413-418

16. Sandy JR, Williams AC, Bearn D, et al. Cleft lip and palate care in the United Kingdom - the Clinical Standards Advisory Group (CSAG) study. Part 1: Background and methodology. Cleft Palate Craniofac J 2001;38(1):20-23. https://doi.org/10.1597/1545-1569_2001_038_0020_clapci_2.0.co_2

17. Semb G, Brattström V, Mølsted K, et al. The Eurocleft study: Intercenter study of treatment outcome in patients with complete cleft lip and palate. Part 1: Introduction and treatment experience. Cleft Palate Craniofac J 2005;42(1):64-68. https://doi.org/10.1597/02-119.1.1

18. Shaw WC, Semb G, Nelson P, et al. The Eurocleft project 1996 - 2000: Overview. J Cranio-Maxillofac Surg 2001;29(3):131-140.
19. Stock NM, Anwar H, Sandy JR, et al. Centralization of cleft lip and palate services in the United Kingdom: The views of adult 'returners'. Cleft Palate Craniofac J 2018;55(5):676-681. https://doi. org/10.1177/1055665617744064

20. Rautio J, Andersen M, Bolund S, et al. Scandcleft randomised trials of primary surgery for unilateral cleft lip and palate: 2. Surgical results. J Plast Surg Hand Surg 2017;51(1):14-20. https://doi.org/10.1080/200 lip and palate: 2 . Sur

21. Semb G, Enemark H, Friede H, et al. A Scandcleft randomised trials of primary surgery for unilateral cleft lip and palate: 1. Planning and management. J Plast Surg Hand Surg 2017;51(1):2-13. https://doi.org/ 656x.2016.1263202

22. Willadsen E, Lohmander A, Persson C, et al. Scandcleft randomised trials of primary surgery for unilateral cleft lip and palate: 5 . Speech outcomes in 5-year-olds - consonant proficiency and errors. J Plast Surg Hand Surg 2017;51(1):38-51. https://doi.org/10.1080/2000656x.2016.1254647

23. Molsted K, Humerinta K, Kuseler A, et al. Scandcleft randomised trials of primary surgery for unilateral cleft lip and palate: 8 . Assessing naso-labial appearance in 5 -year-olds - a preliminary study. J Plast Surg Hand Surg 2017:51(1):64-72. https://doi.org/10.1080/2000656x.2016.1266492

24. Heliovaara A, Kuseler A, Skaare P, et al. Scandcleft randomised trials of primary surgery for unilateral cleft lip and palate: 6. Dental arch relationships in 5 year-olds. J Plast Surg Hand Surg 2017;51(1):52-57. https://doi.org/10.1080/2000656x.2016.1221352

25. Long RE, jun., Hathaway R, Daskalogiannakis J, et al. The Americleft study: An inter-center study of treatment outcomes for patients with unilateral cleft lip and palate. Part 1: Principles and study design Cleft Palate Craniofac J 2011;48(3):239-243. https://doi.org/10.1597/09-180.1

26. Russell K, Long RE, jun., Hathaway R, et al. The Americleft study: An inter-center study of treatment outcomes for patients with unilateral cleft lip and palate. Part 5: General discussion and conclusions. Cleft Palate Craniofac J 2011;48(3):265-270. https://doi.org/10.1597/09-187.1

27. Mars M, Asher-McDade C, Brattström V, et al. A six-center international study of treatment outcome in patients with clefts of the lip and palate. Part 3: Dental arch relationships. Cleft Palate Craniofac 1992;29(5):405-408.

28. Cubitt JJ, Hodges AM, van Lierde KM, et al. Global variation in cleft palate repairs: An analysis of 35219 primary cleft repairs in low- to higher-middle-income countries. Cleft Palate Craniofac J 2014;51(5):553556. https://doi.org/10.1597/12-270

29. Martiniuk AL, Manouchehrian M, Negin JA, et al. Brain gains: A literature review of medical missions to low and middle-income countries. BMC Health Serv Res 2012;12(1):134. https://doi.org/10.1186/14726963-12-134

30. Farmer PE, Kim JY. Surgery and global health: A view from beyond the OR. World J Surg 2008;32(4):533536. https://doi.org/10.1007/s00268-008-9525-9

31. The Smile Train. www.smiletrain.org (accessed 23 April 2019).

32. The Smile Foundation. www.smilefoundationsa.org (accessed 15 April 2019).

33. Rotaplast. www.rotaplast.org (accessed 15 April 2019).

34. Cleft Friends. www.cleftfriends.co.za (accessed 15 April 2019).

35. Lambadusuriya SP, Mars M, Ward CM. Sri Lankan cleft lip and palate project: A preliminary report. J Roy Soc Med 1988;81(12):705-709. https://doi.org/10.1177/014107688808101208

36. Clark JM, Skoner JM, Wang TD. Repair of the unilateral cleft lip/nose deformity. Facial Plast Surg 2003;19(1):29-40. https://doi.org/10.1055/s-2003-39134

37. Ness JA, Sykes JM. Basics of Millard rotation-advancement technique for repair of the unilateral cleft lip deformity. Facial Plast Surg 1993;9(3):167-176. https://doi.org/10.1055/s-2008-1064608

38. De Ladeira PR, Alonso N. Protocols in cleft lip and palate treatment: Systematic review Plast Surg In 2012;2012:562892. https://doi.org/10.1155/2012/562892

39. Penn I, Clayden G, Bentel H. The treatment of cleft lip and palate in South Africa: A review of 500 cases S Afr Med J 1953;27(23):484-488.

40. World Health Organization. Global Strategies to Reduce the Health-care Burden of Craniofacial Anomalies. Geneva: WHO, 2002.

41. Bearn D, Mildinhall S, Murphy T, et al. Cleft lip and palate care in the United Kingdom - the Clinical Standards Advisory Group (CSAG) study. Part 4: Outcome comparisons, training, and conclusions. Cleft Standards Advisory Group (CSAG) study. Part 4: Outcome comparisons, training, and conclusions. Cleft
Palate Craniofac J 2001;38(1):38-43. https://doi.org/10.1597/1545-1569_2001_038_0038_clapci_2.0.co_2

42. Monlleó IL, Gil-da-Silva-Lopes VL. [Craniofacial anomalies: Description and evaluation of treatment Monlleó IL, Gil-da-Silva-Lopes VL. [Craniofacial anomalies: Description and evaluation of treatment
under the Brazilian Unified Health System]. Cadernos de Saúde Pública 2006;22(5):913-922. https://doi. under the Brazilian Unified Heal
org/S0102-311X2006000500004

43. Freitas JAS, Garib DG, Oliveira M, et al. Rehabilitative treatment of cleft lip and palate: Experience of the Hospital for Rehabilitation of Craniofacial Anomalies - USP (HRAC-USP). Part 2: Pediatric dentistry Hospital for Rehabilitation of Craniofacial Anomal
and orthodontics. J Appl Oral Sci 2012;20:268-281.

44. Rispel L. Analysing the progress and fault lines of health sector transformation in South Africa. In Rispel L. Analysing the progress and fault lines of health sector transformation in South Africa. In
Padarath A, King J, Mackie E, Casciola J, eds. South African Health Review 2016. Durban: Health Systems Padarath A,
Trust, 2016.

45. Konst EM, Prahl C, Weersink-Braks H, et al. Cost-effectiveness of infant orthopedic treatment regarding Konst EM, Prahl $\mathrm{C}$, Weersink-Braks $\mathrm{H}$, et al. Cost-effectiveness of infant orthopedic treatment regarding
speech in patients with complete unilateral cleft lip and palate: A randomized three-center trial in the speech in patients with complete unilateral cleft lip and palate: A ra
Netherlands (Dutchcleft). Cleft Palate Craniofac J 2004;41(1):71-77.

46. Masarei A, Wade A, Mars M, et al. A randomized control trial investigating the effect of presurgical orthopedics on feeding in infants with cleft lip and/or palate. Cleft Palate Craniofac J 2007;44(2):182-193. orthopedics on feeding in infants with cleft lip and/or palate. Cleft Palate Craniofac J 2007;44(2):182-193.
Bessell A, Hooper L, Shaw WC, et al. Feeding interventions for growth and development in infants with Bessell A, Hooper L, Shaw WC, et al. Feeding interventions for growth and development in infants with
cleft lip, cleft palate or cleft lip and palate. Cochrane Database Syst Rev 2011;16;(2):CD003315. https:// cleft lip, cleft palate or cleft lip and palate. C
doi.org/10.1002/14651858.CD003315.pub3

doi.org/10.1002/14651858.CD003315.pub3
48. Hlongwa P, Rispel LC. 'People look and ask lots of questions': Caregivers' perceptions of healthcare Hlongwa P, Rispel LC. 'People look and ask lots of questions': Caregivers' perceptions of healthcare
provision and support for children born with cleft lip and palate. BMC Publ Health 2018;18(1):506. provision and support for children born w
https://doi.org/10.1186/s12889-018-5421-x

49. Eshete M, Butali A, Deressa W, et al. Descriptive epidemiology of orofacial clefts in Ethiopia. J Craniofac Surg 2017;28(2):334-337. https://doi.org/10.1097/scs.0000000000003234

50. Tollefson TT, Shaye D, Durbin-Johnson B, et al. Cleft lip-cleft palate in Zimbabwe: Estimating the distribution of the surgical burden of disease using geographic information systems. Laryngoscope 2015;125(Suppl 1):S1-S14. https://doi.org/10.1002/lary.24747

51. Nagalo K, Ouédraogo I, Laberge J-M, et al. Epidemiology, clinical aspects and management of cleft lip and/or palate in Burkina Faso: A humanitarian pediatric surgery-based study. Open J Pediat 2015;5(2):113-120. https://doi.org/10.4236/ojped.2015.52017

52. Chiu L, Kanth A, Adetayo OA. Social interactions of school-aged children with craniofacial anomalies: Mothers' perspectives and advice. Cleft Palate Craniofac J 2016;53(5):622-623. https://doi org/10.1597/15-113

3. Mossey PA, Modell B. Epidemiology of oral clefts 2012: An international perspective. Front Oral Biol 2012;16:1-18. https://doi.org/10.1159/000337464

4. Johansson B, Ringsberg KC. Parents' experiences of having a child with cleft lip and palate. J Adv Nurs 2004;47(2):165-173

55. Turner SR, Thomas PWN, Dowell T, et al. Psychological outcomes amongst cleft patients and their families. Br J Plast Surg 1997;50(1):1-9. https://doi.org/https://doi.org/10.1016/S0007-1226(97)91275-3

56. Pannbacker M, Scheuerle J. Parents' attitudes toward family involvement in cleft palate treatment. Cleft Palate Craniofac J 1993;30(1):87-89. https://doi.org/10.1597/1545-1569_1993_030_0087_patfii_2.3.co_2

Accepted 4 December 2018 